\title{
Segmental Kaposi Sarcoma in an Immunocompromised Patient
}

\author{
Lori S. Kim, BA ${ }^{1}$, Erin Dodd, MD², Angad A. Chadha, MD² \\ ${ }^{1}$ University of Illinois at Chicago College of Medicine, Chicago, IL \\ ${ }^{2}$ Section of Dermatology, University of Chicago, Chicago, IL
}

\section{ABSTRACT}

Kaposi sarcoma (KS) is a vascular tumor that classically presents as multiple violaceous lesions on the extremities in a widespread manner. A 60-year-old male with a history of immunosuppression in the setting of bilateral lung transplant presented with numerous violaceous papulonodules and patches localized on the left flank, with a biopsy confirming the diagnosis of KS. We present a rare case of KS presenting in a segmental distribution and review the available literature with a discussion of the possible mechanisms behind segmental and dermatomal KS.

\section{INTRODUCTION}

Kaposi's sarcoma (KS) is a low-grade vascular tumor that has been reported with frequency in immunocompromised patients. There are four clinical variants: classical, endemic (or African), AIDS-associated, and iatrogenic immunosuppression-associated $\mathrm{KS} .{ }^{1}$ Although the clinical presentation of KS varies amongst these clinical variants, KS typically presents with violaceous macules, papules, and plaques on the bilateral lower extremities, face, and oral mucosa. ${ }^{2}$ Extracutaneous manifestations most often present in the gastrointestinal tract and respiratory system It is exceedingly rare for KS to be localized to a solitary body segment that is not located on the lower extremities. We report a case of an immunocompromised organ transplant patient who developed KS in a segmental distribution of his left flank.

\section{CASE PRESENTATION}

The inpatient dermatology consult service was consulted on a 60-year-old Caucasian male with a history of a bilateral lung transplant, now presenting with a rash on the left abdomen that started a few weeks prior. He denied any pain or pruritus and denied any similar rashes in the past. He did not report any rash anywhere else on his body, including any oral or genital lesions. His contributory medications included posttransplant immunosuppression with azathioprine, tacrolimus, and prednisone.

On exam, the patient had numerous nontender, non-blanching, violaceous ovoid papulonodules and patches scattered only on the left lateral abdomen with few ecchymoses (Figure 1). A skin biopsy of one of the papulonodules showed a spindle cell proliferation in the dermis forming sheets and fascicles with slit-like spaces filled with red blood cells, compatible with a diagnosis of Kaposi Sarcoma. Human herpesvirus 8 
immunostaining was positive in the neoplastic cells. Fecal occult blood testing was negative. Chest $x$-ray at that time demonstrated widespread interstitial edema and bilateral pleural effusions thought to be due to volume overload but the possibility of pulmonary KS was not radiographically ruled out.

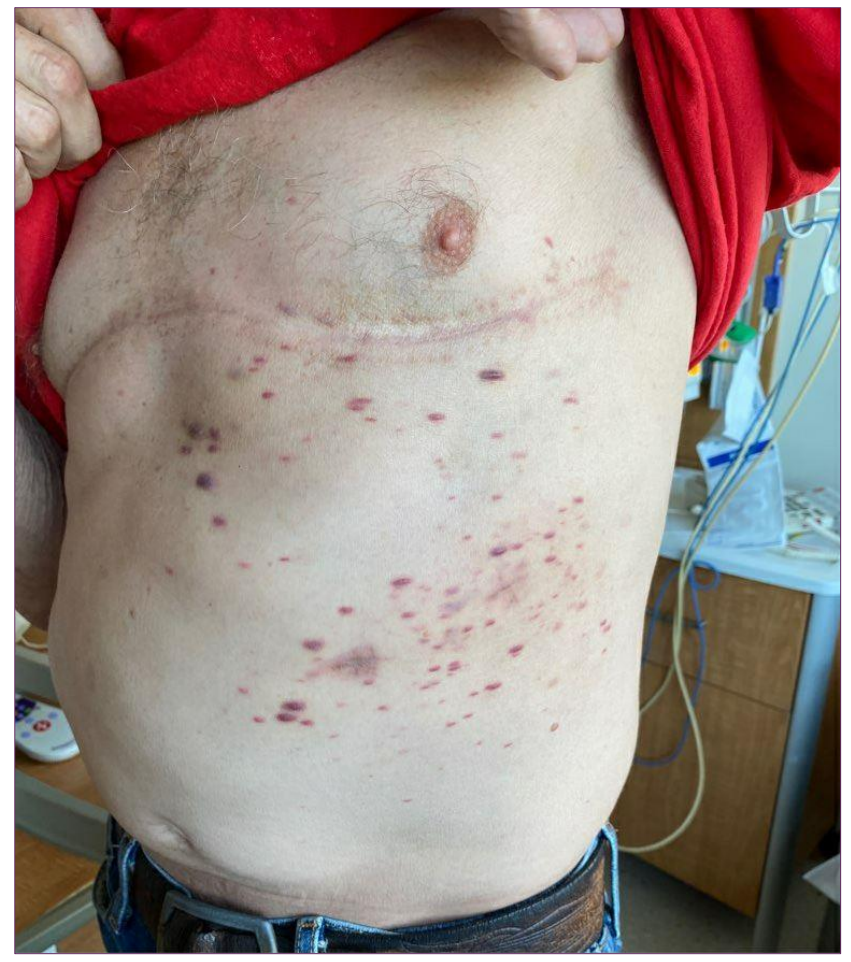

Figure 1

The patient was not a candidate for local treatments alone due to the number of lesions; he was originally discharged with plans to adjust his immunosuppression and assess KS response before considering local therapies. Outpatient PET/CT was to be pursued. However, 3 weeks later the patient was readmitted for type 1 respiratory failure requiring intubation after a routine bronchoscopy. At that time, a CT scan of the chest revealed bilateral ground-glass pulmonary opacities with moderate pleural effusions; this was thought to likely be multifactorial due to pneumonia and possibly rapidly progressing KS. Given the patient's tenuous clinical status and difficulty obtaining a lung biopsy, he was treated empirically with broad-spectrum antimicrobials and soon thereafter with intravenous liposomal doxorubicin for possible KS. Eventually, due to lack of clinical response, trans-bronchial lung biopsy was pursued and revealed acute fibrinous and organizing pneumonia without evidence of KS in the lungs. Soon after the biopsy, the patient was placed on continuous veno-venous hemodialysis (CVVHD) due to severe acute kidney injury and thereafter placed on veno-venous extra corporeal membrane oxygenation (ECMO) due to declining lung function. The patient's family ultimately decided to transition the patient to comfort care; he expired shortly thereafter.

\section{DISCUSSION}

Here we present a case of segmental KS in a patient on iatrogenic immunosuppression after lung transplant. Given that KS is more often found on the legs and face, this is a relatively rare presentation of this disease.

A dermatomal presentation of KS was first documented by Niedt, who presented a patient with a history of intravenous drug use who developed herpes zoster infection in the left T3 distribution and then two months lateral developed KS in the same distribution. ${ }^{4}$ Niedt proposed that this presentation was due to the Koebner phenomenon, which is when a previously present disease recurs in a site of trauma. ${ }^{4}$

The Koebner phenomenon was further elucidated by Wolf, who coined the term Wolf's isotopic response. ${ }^{5}$ Whereas Koebner's isomorphic phenomenon describes the appearance of a skin lesion that is morphologically similar to an existing skin disease at a site of injury, Wolf's 
isotopic response describes the occurrence of a new, unrelated disease that appears at the same site of a previously healed disease. ${ }^{5}$ Numerous postherpetic isotopic responses have been documented, including but not limited to granuloma annulare, breast carcinoma, leukemic infiltration, and lichen planus; however, KS is extremely rare in that the case report by Niedt is the only documented so far. $4,6,7$

Our patient's presentation cannot quite be explained by either the Koebner phenomenon or Wolf's isotopic response, as he did not report a previous history of herpes zoster virus (HZV) or other rashes at the site of his KS. A similar case was reported by Eisman, who documented a patient presenting with KS on his right lower leg and right chest in a T5 dermatone distribution without any history of previous $\mathrm{HZV}{ }^{8}$ One of the mechanisms he proposed was "locus minoris resistentiae" which describes eczematous eruptions that develop in areas of previously injured skin, broadly including any history of surgery, trauma, or scar. ${ }^{9}$ This certainly might be possible for our patient who had a bilateral lung transplant but would be unusual as the KS presented more on his flank rather than the site of surgery.

The review article on the postherpetic isotopic response by Ruocco describes several possible pathogenetic mechanisms of Wolf's isotopic response including viral, immunologic, vascular, and neural origin. ${ }^{6}$ Of these, the neural etiology is of particular interest as it hypothesizes that underlying neurologic alteration with consequent immunologic impairment is the first step in the isotopic response. ${ }^{6}$ This consequently raises the possibility of KS appearing as Wolf's isotopic response within an area previously affected by "zoster sine herpete" which could have occurred sub-clinically without the patient's knowledge. This proposed relationship between neurologic alteration, immunology, and the skin may give us a better insight into our patient's presentation, given his segmental KS presentation and his history of immunosuppression.

\section{CONCLUSION}

We present a case of an immunosuppressed patient who developed KS in an unusual segmental distribution. Additionally, we provide a literature review of the limited reported cases of segmental and dermatomal KS, with emphasis placed on the possible pathophysiology behind this presentation.

\section{Conflict of Interest Disclosures: None}

Funding: None

Corresponding Author:

Angad A Chadha, MD

5841 S Maryland Ave

MC 5067

Chicago, IL 60637

Phone 773-702-1611

Fax 773-834-7071

Email: chadha@uchicago.edu

\section{References:}

1. Fitzpatrick's Dermatology, 9e | AccessMedicine | McGraw-Hill Medical. Accessed March 16, 2021. https://accessmedicine.mhmedical.com/book.asp $x$ ?bookID=2570

2. Radu O, Pantanowitz L. Kaposi Sarcoma. Archives of Pathology \& Laboratory Medicine. 2013;137(2):289-294. doi:10.5858/arpa.20120101-RS

3. Beasley MB, Franks TJ, Galvin JR, Gochuico B, Travis WD. Acute fibrinous and organizing pneumonia: a histological pattern of lung injury and possible variant of diffuse alveolar damage. Arch Pathol Lab Med. 2002;126(9):1064-1070. doi:10.1043/00039985(2002)126<1064:AFAOP>2.0.CO;2

4. William Niedt G, Prioleau PG. Kaposi's sarcoma occurring in a dermatome previously involved by January 2022 Volume 6 Issue 1 
herpes zoster. Journal of the American Academy of Dermatology. 1988;18(2):448-451. doi:10.1016/S0190-9622(88)70068-7

5. Wolf R, Brenner S, Ruocco V, Filioli FG. ISOTOPIC RESPONSE. Int J Dermatol. 1995;34(5):341-348. doi:10.1111/j.13654362.1995.tb03616.x

6. Ruoccoa V, Ruoccoa E, Ghersetichb I, Bianchib $\mathrm{B}$, Lottib T. Isotopic response after herpesvirus infection: An update. Journal of the American Academy of Dermatology. 2002;46(1):90-94. doi:10.1067/mjd.2002.118362

7. Wang $T$, Zhang $M$, Zhang $Y$, et al. Wolf's Isotopic Response after Herpes Zoster Infection: A Study of 24 New Cases and Literature Review. Acta Derm Venerol. 2019;99(11):953-959. doi:10.2340/00015555-3269

8. Eisman S, O'Toole EA, Collis C, Rustin MHA. Zosteriform Kaposi's sarcoma. Clinical and Experimental Dermatology. 2001;26(5):402-404. doi:https://doi.org/10.1046/j.13652230.2001.00845.x

9. Dermatitis in loco minoris resistentiae. Journal of the American Academy of Dermatology. 1982;6(6):1010-1013. doi:10.1016/S01909622(82)80099-6 